\title{
Elastic Scattering of Halo Nuclei
}

\author{
R. C. Johnson, J. S. Al-Khalili, and J.A. Tostevin \\ Department of Physics, University of Surrey, Guildford, Surrey, GU2 5XH, United Kingdom
}

(Received 24 March 1997)

\begin{abstract}
We show that under certain conditions a simple relationship exists between the elastic scattering of a composite halo nucleus and of its core from a stable target. The coupling of the elastic and projectile excitation channels is crucial to the analysis, which is particularly useful when the ratio of the halo to the core mass is small. In the case of ${ }^{11} \mathrm{Be}$ elastic scattering the cross section relationship is quite well satisfied. For both ${ }^{11} \mathrm{Be}$ and ${ }^{19} \mathrm{C}$ our analysis reveals a significant sensitivity of elastic scattering data to the halo size and structure. [S0031-9007(97)03995-1]
\end{abstract}

PACS numbers: 25.60.Bx, 24.10.-i, 25.70.Bc

The existence of a class of light nuclei with a localized central core surrounded by a dilute "halo" of neutron matter is now well established. Evidence for these novel structures has been gained mainly from measurements of total neutron removal [1] and breakup reaction cross sections [2-4], particularly at high energy. We investigate to what extent complementary information can be gained from high quality elastic scattering measurements at lower energies.

In this Letter we show that, in certain circumstances, the elastic scattering of a halo nucleus from a stable target can give simple direct evidence for the structure of the halo. The theory makes explicit use of the characteristics of halo nuclei, namely their very small neutron separation energy and the large spatial extension of the halo, which in turn result in strong coupling between the halo ground state and low energy excitations. This coupling of the elastic and projectile excitation channels plays a crucial role in the analysis, the results of which cannot be readily understood in terms of optical or folding model approaches. In elastic scattering, the analysis is expected to be particularly useful in systems where the ratio of the halo particle mass to the core mass is small.

We consider the scattering of an assumed two-body projectile nucleus, composed of a core of mass $m_{c}$ and a valence particle of mass $m_{v}$, by a third (target) nucleus of mass $m_{T}$. It is assumed that these three bodies are spinless and structureless, although these are not essential assumptions. Two key conditions must be met, however, for the subsequent development to be a useful one:

(i) The interaction between the core and target $\left(V_{c T}\right)$ should be effectively much stronger than that between the valence and target particles $\left(V_{v T}\right)$.

(ii) The relative motion of the core and valence particles is slow compared to the relative motion of the center of mass of the projectile and target, and so can be treated adiabatically, in the spirit to the work of Johnson and Soper [5].

Requirement (i) places limitations on the likely regions of applicability of our model. In strong interaction- dominated processes, it is likely to be most valid when the number of nucleons in the core far exceeds that of the valence body. For ${ }^{11} \mathrm{Be}$ elastic scattering, where this ratio is $10: 1$, the assumption will be seen to be a good starting point for small scattering angles. Requirement (i) could also be approached in Coulomb dominated processes when the core is charged and the valence particle is neutral, as is typical in halo nuclei. These Coulomb dominated processes will be considered elsewhere. Condition (ii) is expected to be satisfied for sufficiently high incident energy and is consistent with the weak binding of halo nuclei.

We first show how assumptions (i) and (ii), without further approximations, lead to a very transparent relationship between the elastic scattering differential cross sections of (a) the composite two-body halo projectile, and (b) its core, from the target nucleus.

In the absence of the valence particle-target interaction $\left(V_{v T}=0\right)$, the equation satisfied by the three-body scattering wave function $\Psi_{\boldsymbol{K}}^{(+)}(\boldsymbol{r}, \boldsymbol{R})$, corresponding to the incident projectile with momentum $\boldsymbol{K}$ in the projectile-target center of mass (c.m.) frame, is

$$
\left[T_{\boldsymbol{R}}+V_{c T}\left(\boldsymbol{R}-\alpha_{v c} \boldsymbol{r}\right)+H_{v c}-E\right] \Psi_{\boldsymbol{K}}^{(+)}(\boldsymbol{r}, \boldsymbol{R})=0 .
$$

Here $H_{v c}$ is the internal Hamiltonian for the valence-core system, $\boldsymbol{R}$ is the projectile-target separation, with $T_{\boldsymbol{R}}$ the corresponding kinetic energy operator, and $\boldsymbol{r}$ is the core to valence particle relative coordinate. The quantity $\alpha_{v c}=$ $m_{v} /\left(m_{v}+m_{c}\right)$, and $\boldsymbol{R}_{c T}=\boldsymbol{R}-\alpha_{v c} \boldsymbol{r}$ is the core-target separation vector. The wave function $\Psi_{\boldsymbol{K}}^{(+)}(\boldsymbol{r}, \boldsymbol{R})$ satisfies scattering boundary conditions of the form

$$
\Psi_{\boldsymbol{K}}^{(+)}(\boldsymbol{r}, \boldsymbol{R})=\phi_{0}(\boldsymbol{r}) e^{i \boldsymbol{K} \cdot \boldsymbol{R}}+\text { outgoing waves, }
$$

where the outgoing waves describe elastic scattering and excitations of the projectile. The ground state wave function of the projectile $\phi_{0}(\boldsymbol{r})$ satisfies $H_{v c} \phi_{0}(\boldsymbol{r})=$ $-\varepsilon_{0} \phi_{0}(\boldsymbol{r})$.

The adiabatic assumption (ii) means that we can replace $H_{v c}$ in Eq. (1) by a constant. This is chosen to be $-\varepsilon_{0}$. 
The corresponding approximate three-body wave function $\Psi_{K}^{(+) \text {Ad }}$ now satisfies

$$
\left[T_{\boldsymbol{R}}+V_{c T}\left(\boldsymbol{R}-\alpha_{v c} \boldsymbol{r}\right)-E_{0}\right] \Psi_{\boldsymbol{K}}^{(+) \mathrm{Ad}}(\boldsymbol{r}, \boldsymbol{R})=0,
$$

where $E_{0}=E+\varepsilon_{0}=\hbar^{2} K^{2} / 2 \mu$ is the incident c.m. kinetic energy and $\mu$ is the projectile-target reduced mass. A consequence of the adiabatic assumption is that $r$ is now just a parameter in Eq. (3) and, because $V_{v T}=0, \boldsymbol{R}$ and $\boldsymbol{r}$ appear only in the combination $\boldsymbol{R}^{\prime}=\boldsymbol{R}_{c T}=\boldsymbol{R}-$ $\alpha_{v c} \boldsymbol{r}$. Equation (3) is therefore simplified by transforming to this variable. A solution of Eq. (3) is therefore $\chi_{\boldsymbol{K}}^{(+)}\left(\boldsymbol{R}^{\prime}\right)$ multiplied by an arbitrary function of $\boldsymbol{r}$, where $\chi_{\boldsymbol{K}}^{(+)}$satisfies the Schrödinger equation

$$
\left[T_{\boldsymbol{R}^{\prime}}+V_{c T}\left(\boldsymbol{R}^{\prime}\right)-E_{0}\right] \chi_{\boldsymbol{K}}^{(+)}\left(\boldsymbol{R}^{\prime}\right)=0,
$$

with outgoing wave boundary conditions. Choosing the multiplicative function so that the adiabatic wave function satisfies Eq. (2) as well as Eq. (3) gives [6,7]

$$
\Psi_{\boldsymbol{K}}^{(+) \mathrm{Ad}}(\boldsymbol{r}, \boldsymbol{R})=\phi_{0}(\boldsymbol{r}) e^{i \alpha_{v c} \boldsymbol{K} \cdot \boldsymbol{r}} \chi_{\boldsymbol{K}}^{(+)}\left(\boldsymbol{R}^{\prime}\right),
$$

Clearly $\chi_{\boldsymbol{K}}^{(+)}$describes the scattering of a particle of mass $\mu$ from the potential $V_{c T}$ and corresponds to a model in which the projectile is pointlike. We emphasize that the three-body wave function, Eq. (5), includes breakup components and excitations to any bound states as is clear from its complicated dependence on $\boldsymbol{r}$, through the argument $\boldsymbol{R}^{\prime}$ of $\chi_{\boldsymbol{K}}^{(+)}$and the exponential factor $\exp \left(i \alpha_{v c} \boldsymbol{K} \cdot \boldsymbol{r}\right)$.

The elastic scattering transition amplitude for the projectile, from initial state $\boldsymbol{K}$ into final state $\boldsymbol{K}^{\prime}$, is

$$
\begin{aligned}
T_{\mathrm{el}}\left(\boldsymbol{K}^{\prime}, \boldsymbol{K}\right)= & \int d \boldsymbol{r} \int d \boldsymbol{R} \phi_{0}^{*}(\boldsymbol{r}) e^{-i \boldsymbol{K}^{\prime} \cdot \boldsymbol{R}} \\
& \times V_{c T}\left(\boldsymbol{R}-\alpha_{v c} \boldsymbol{r}\right) \Psi_{\boldsymbol{K}}^{(+)}(\boldsymbol{r}, \boldsymbol{R}) .
\end{aligned}
$$

Using the adiabatic approximation to $\Psi_{\boldsymbol{K}}^{(+)}$, Eq. (5), and making the change of variable from $\boldsymbol{R}$ to $\boldsymbol{R}^{\prime}$ this factorizes as

$$
\begin{aligned}
T_{\mathrm{el}}\left(\boldsymbol{K}^{\prime}, \boldsymbol{K}\right)= & {\left[\int d \boldsymbol{r}\left|\phi_{0}(\boldsymbol{r})\right|^{2} e^{i \alpha_{v c}\left(\boldsymbol{K}-\boldsymbol{K}^{\prime}\right) \cdot \boldsymbol{r}}\right] } \\
& \times\left[\int d \boldsymbol{R}^{\prime} e^{-i \boldsymbol{K}^{\prime} \cdot \boldsymbol{R}^{\prime}} V_{c T}\left(\boldsymbol{R}^{\prime}\right) \chi_{\boldsymbol{K}}^{(+)}\left(\boldsymbol{R}^{\prime}\right)\right] .
\end{aligned}
$$

The same result is obtained by examining the asymptotic form of Eq. (5) in the elastic channel. The second integral here is just the transition amplitude $T_{\mathrm{pt}}\left(\boldsymbol{K}^{\prime}, \boldsymbol{K}\right)$ for a point projectile scattering from the core-target potential $V_{c T}$. Thus, the effects of projectile excitation and structure arise entirely through the first integral, the form factor

$$
F(\boldsymbol{Q})=\int d \boldsymbol{r}\left|\phi_{0}(\boldsymbol{r})\right|^{2} \exp (i \boldsymbol{Q} \cdot \boldsymbol{r}),
$$

where $\boldsymbol{Q}=\alpha_{v c}\left(\boldsymbol{K}-\boldsymbol{K}^{\prime}\right)$. $\mathbf{Q}$ can be identified with the momentum change suffered by the valence particle in the scattering event (ignoring small binding effects).

The corresponding elastic scattering differential cross section is therefore

$$
\left(\frac{d \sigma}{d \Omega}\right)_{\mathrm{el}}=|F(\boldsymbol{Q})|^{2}\left(\frac{d \sigma}{d \Omega}\right)_{\mathrm{pt}},
$$

where $(d \sigma / d \Omega)_{\mathrm{pt}}$ is the cross section for a point projectile, with mass $\mu$, scattering by the core-target interaction. This quantity is therefore very closely related to the experimental projectile core-target elastic scattering. The importance of Eq. (8) is that it clarifies the relevant scattering angles and incident energies at which a halo, of a given size and structure, will be manifest as a deviation from the scattering due to a point projectile.

Equation (9) is reminiscent of factorizations which occur in electron scattering when using Born approximation and approximate distorted wave theories. Note, however, that the present analysis does not involve Born approximation in any sense, and only if all intermediate states are included do the second and higher order terms in the Born series factorize in this way. The same argument obtains for the factorization of the wave function in Eq. (5).

An alternative derivation of Eqs. (5) and (9) based on an integral equation formulation will be presented elsewhere [6]. In the same reference we also examine the validity of the adiabatic approximation (ii) when assumption (i) is also valid.

We present form factors $|F(Q)|^{2}$ and cross section angular distributions for ${ }^{11} \mathrm{Be}+{ }^{12} \mathrm{C}$ and ${ }^{19} \mathrm{C}+{ }^{12} \mathrm{C}$ elastic scattering, effective three-body ${ }^{10} \mathrm{Be}+n+{ }^{12} \mathrm{C}$ and ${ }^{18} \mathrm{C}+n+{ }^{12} \mathrm{C}$ systems. ${ }^{11} \mathrm{Be}$ is a good example of a binary, ${ }^{10} \mathrm{Be}+n$, single neutron halo nucleus and ${ }^{19} \mathrm{C}$ is also a single neutron halo candidate [8]. Both systems have small $m_{v} / m_{c}$ ratios. For ${ }^{11} \mathrm{Be}+{ }^{12} \mathrm{C}$, there are preliminary small angle elastic scattering data [9] for both the ${ }^{10} \mathrm{Be}$ core and the ${ }^{11} \mathrm{Be}$ composite, but at energies of 59.4 and $49.3 \mathrm{MeV} / A$, respectively. Ideally these data are required at the same energy per nucleon to provide the necessary information on $V_{c T}$, which is an essential ingredient in the context of Eq. (9).

For ${ }^{11} \mathrm{Be}$ the wave functions were taken to be pure $2 s_{1 / 2}$ neutron single particle states, with separation energy $0.503 \mathrm{MeV}$, calculated in a central Wood-Saxon potential [10]. By changing the binding potential geometry, we generate ${ }^{11} \mathrm{Be}$ composites with different rms matter radii and hence $|F(Q)|^{2}$. For ${ }^{19} \mathrm{C}$ the ground state structure is presently uncertain with speculations of it being a pure $2 s_{1 / 2}$ state, $1 d_{5 / 2}$ state, or a linear combination of such configurations [11]. The neutron separation energy was $0.240 \mathrm{MeV}$.

According to Eq. (9) the form factor $|F(Q)|^{2}$, which multiplies the point particle cross section, reflects the modifications to the scattering due to the composite nature of the projectile. In Fig. 1 we first show these calculated $|F(Q)|^{2}$, as a function of c.m. angle, appropriate for the elastic scattering of ${ }^{11} \mathrm{Be}$ (upper part) and ${ }^{19} \mathrm{C}$ (lower part) from ${ }^{12} \mathrm{C}$ at 49.3 and $30 \mathrm{MeV} / A$, respectively. These energies are relevant to recent experiments with these beams. These calculations demonstrate clearly the sensitivity of the formfactor to the halo properties. 


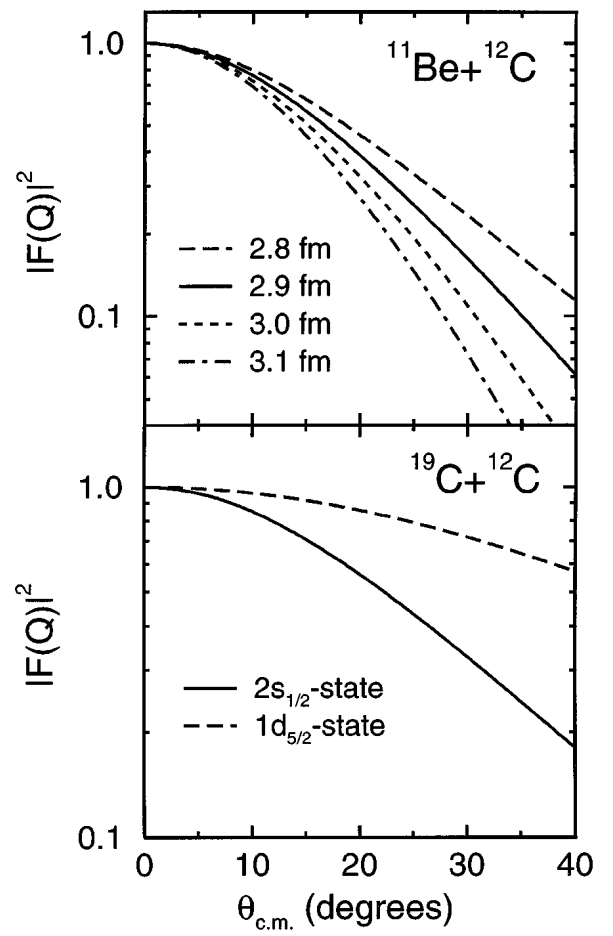

FIG. 1. Calculated $|F(Q)|^{2}$, as a function of c.m. scattering angle for the elastic scattering of ${ }^{11} \mathrm{Be}$ (upper part) and ${ }^{19} \mathrm{C}$ (lower part) from ${ }^{12} \mathrm{C}$ at 49.3 and $30 \mathrm{MeV} / A$, respectively.

Conversely, they reflect the information available from halo nucleus elastic scattering data on the halo-core relative motion wave function. For ${ }^{11} \mathrm{Be}$, the halo is seen to result in a reduction in the elastic differential cross section by a factor of between 2 and 4 at $20^{\circ}$, compared to that for point particle scattering. There is also a significant sensitivity to the assumed rms separation of the valence and core particles. For ${ }^{19} \mathrm{C}$, the $|F(Q)|^{2}$ which result from a pure $2 s_{1 / 2}$ (solid curve) or $1 d_{5 / 2}$ (dashed curve) neutron state are shown. The departures from point particle scattering are predicted to be significantly different for a $2 s_{1 / 2}$ and $1 d_{5 / 2}$ valence neutron, with almost a factor of 2 difference in the cross sections at $20^{\circ}$. We note that, although the leading term in the expansion of the formfactor about $Q=0$ gives a deviation from unity proportional to the mean squared separation of the core and valence particles in the projectile, the values of $Q$ which enter in the examples above are such that this leading order term is inadequate and there is sensitivity in the $F(Q)$ to higher order moments, except at the very smallest angles.

These conclusions, and the factorization into a point particle cross section and formfactor in Eq. (9), assume the dominance of $V_{c T}$, our assumption (i). For such three-body systems full quantum mechanical calculations, which make the adiabatic approximation, assumption (ii), but which do not make the additional assumption (i), concerning the dominance of $V_{c T}$, can also be performed [12]. These use the ${ }^{10} \mathrm{Be}+{ }^{12} \mathrm{C}$ optical potential $V_{c T}$ of [10], which is consistent with the available data at $59.4 \mathrm{MeV} / A$. When included, the valence particle-target, i.e., neutron $+{ }^{12} \mathrm{C}$, optical potential $V_{v T}$ is that tabulated in [13].

Figure 2 shows the calculated elastic differential cross section angular distributions (ratio to Rutherford) for ${ }^{11} \mathrm{Be}+{ }^{12} \mathrm{C}$ scattering at $49.3 \mathrm{MeV} / A$. The dashed curve shows the point projectile differential cross section $(d \sigma / d \Omega)_{\mathrm{pt}}$ calculated using the core-target potential. The dot-dashed curve shows the calculated cross section in the no excitation limit, which means using the folding model interaction for the projectile. The similarity of the folding and point particle calculations makes clear that the effects associated simply with folding the core and valence particle interactions over the size of the halo are relatively minor, and that the multiplicative form factor, shown by the short dashed line (the $2.9 \mathrm{fm}$ rms case of Fig. 1) is associated principally with the large projectile excitation effects. The product of the point cross section and formfactor, the calculation making assumptions (i) and (ii), produces the solid line, which is to be compared with the full adiabatic model calculation [assumption (ii) only], including $V_{n T}$, shown by the full circle symbols. The agreement is reasonable and suggests the dominance of $V_{c T}$, although the effects of the valence neutron-target interaction are not negligible.

We observe therefore that halo nucleus elastic scattering angular distributions are strongly affected by projectile excitation channels and the spatial size of the halo. However, these effects are principally manifest simply through a multiplicative formfactor, dependent only on the halo ground state wave function and which multiplies the cross section due to point particle scattering by the interaction due to

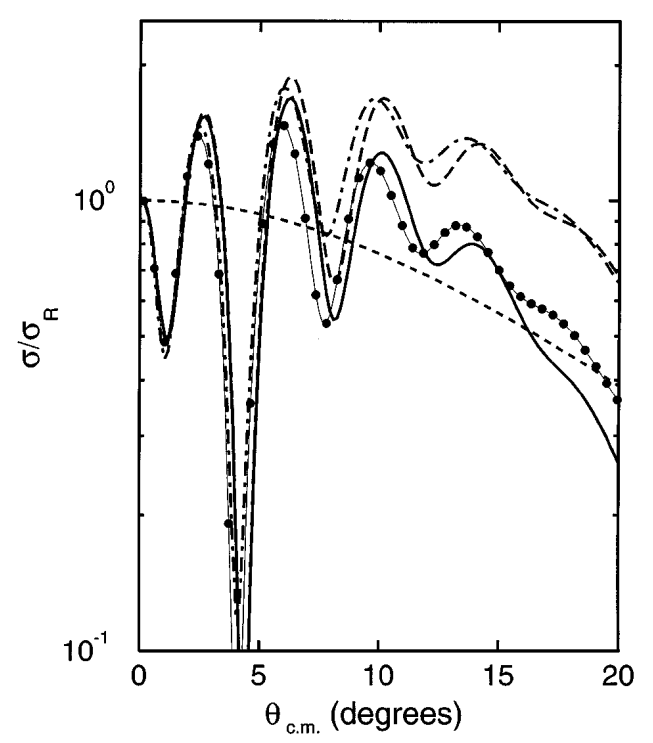

FIG. 2. Calculated elastic differential cross section angular distributions (ratio to Rutherford) for ${ }^{11} \mathrm{Be}+{ }^{12} \mathrm{C}$ scattering at $49.3 \mathrm{MeV} / A$. The curves are discussed in the text. 


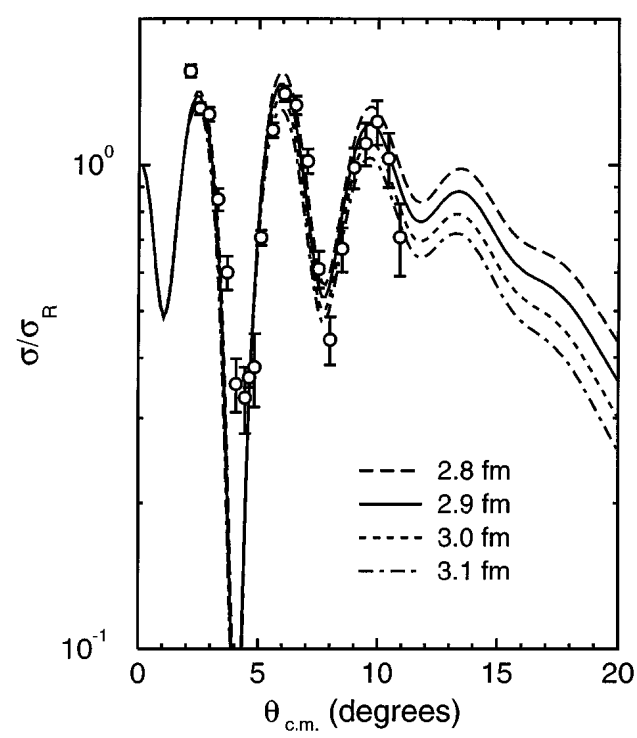

FIG. 3. Calculated elastic differential cross section angular distributions (ratio to Rutherford) for ${ }^{11} \mathrm{Be}+{ }^{12} \mathrm{C}$ scattering at $49.3 \mathrm{MeV} / A$ calculated using the adiabatic approximation and including the neutron-target interaction.

the core. The latter can be highly constrained empirically if high quality data for the core system are also available. In final quantitative studies, it may be important to include also the interaction of the valence particle. This is not a difficulty since exact adiabatic calculations, without the additional assumption (i), are possible for both two- and three-body projectile systems $[12,14]$. In Fig. 3 we show the results of such adiabatic calculations for ${ }^{11} \mathrm{Be}+{ }^{12} \mathrm{C}$ scattering at $49.3 \mathrm{MeV} / A$. These include the neutron target interaction and correspond to the four ${ }^{11} \mathrm{Be}$ wave functions with different rms radii discussed in connection with Fig. 1. The data are from [9]. We note that the behavior of the cross sections expected on the basis of the formfactors of Fig. 1, and the insight they provide, remain clearly evident and that data are now reaching a quality where elastic scattering data could indeed yield independent information on halo structures.
The financial support of the United Kingdom Engineering and Physical Sciences Research Council (EPSRC) in the form of Grant No. GR/J95867 is gratefully acknowledged.

[1] T. Kobayashi et al., Phys. Lett. B 232, 51 (1989).

[2] I. Tanihata et al., Phys. Lett. B 160, 380 (1985).

[3] I. Tanihata, T. Kobayashi, O. Yamakawa, T. Shimoura, K. Ekuni, K. Sugimoto, N. Takahashi, T. Shimoda, and H. Sato, Phys. Lett. B 206, 592 (1988).

[4] W. Mittig et al., Phys. Rev. Lett. 59, 1889 (1987).

[5] R. C. Johnson and P. J.R. Soper, Phys. Rev. C 1, 976 (1970).

[6] R. C. Johnson, "Elastic scattering and elastic break-up of halo nuclei in a special model," Proceedings of European Conference on Advances in Nuclear Physics and Related Areas, Thessaloniki, Greece, 8-12 July 1997 (to be published).

[7] These simplifications to the three-body wave function resulting from assumptions (i) and (ii) were noted previously [R. C. Johnson, M. Kamimura, and M. Yahiro, private communication, quoted in M. Kamimura, Muon Catalysed Fusion 1, 333 (1987)] and were used there in a different context.

[8] D. Bazin et al., Phys. Rev. Lett. 74, 3569 (1995); F. M. Marqués et al., Phys. Lett. B 381, 407 (1996).

[9] P. Roussel-Chomaz, private communication.

[10] J. S. Al-Khalili, J. A. Tostevin, and J. M. Brooke, Phys. Rev. C 55, R1018 (1997).

[11] D. Ridikas, M.H. Smedberg, J.S. Vaagen, and M. V. Zhukov, Europhys. Lett. 37, 385 (1997).

[12] H. Amakawa, S. Yamaji, A. Mori, and K. Yazaki, Phys. Lett. B 82, 13 (1979); I. J. Thompson, Computer program ADIA, Daresbury Laboratory Report, 1984, unpublished.

[13] J. S. Al-Khalili, I. J. Thompson, and J. A. Tostevin, Nucl. Phys. A581, 331 (1995).

[14] J. A. Christley, J. S. Al-Khalili, J. A. Tostevin, and R. C. Johnson, Nucl. Phys. A624, 275 (1997). 